\title{
Does CDW Physics Allow Ultra Fast Transitions, and Current vs. Applied Electric Field Values as Seen in Alaboratory Setting?
}

\author{
Andrew Walcott Beckwith \\ Department of Physics, Chongqing University, Chongqing, China \\ Email: rwill9955b@gmail.com
}

Received 22 April 2014; revised 15 May 2014; accepted 21 May 2014

Copyright (C) 2014 by author and Scientific Research Publishing Inc.

This work is licensed under the Creative Commons Attribution International License (CC BY).

http://creativecommons.org/licenses/by/4.0/

(c) (i) Open Access

\begin{abstract}
We reference the tunneling Hamiltonian to have particle tunneling among different states represented as wave-functions. Our problem applies wave-functionals to a driven sine-Gordon system. We apply the tunneling Hamiltonian to charge density wave (CDW) transport problems where we consider tunneling among states that are wave-functionals of a scalar quantum field, i.e. derived I-E curves that match Zenier curves used to fit data experimentally with wave-functionals congruent with the false vacuum hypothesis. The open question is whether the coefficients picked in both wave-functionals and the magnitude of the coefficients of the driven sine-Gordon physical system are picked by topological charge arguments that appear to assign values consistent with the false vacuum hypothesis. Crucial results by Fred Cooper et al. allow a mature quantum foam interpretation of false vacuum nucleation for further refinement of our wave-functional results. In doing so, we give credence to topological arguments as a first order phase transition in CDW I-E curves.
\end{abstract}

\section{Keywords}

Driven Sine Gordon Physical System, CDW, Topological Charge, False Vacuum Hypothesis, I-E Curves

\section{Introduction}

This document allows for comparison of results derived by the author which are improving on both the Zener plots and Lin's generalization of Schwingers 1950 electron-positron nucleation values results for low dimensional systems as cited in references [1] [2]. In doing so, we cite results derived by the author [1] [2] including 
having a threshold electric field explicitly as a starting point without an arbitrary cut off value for the start of the graphed results. We conclude with a discussion of how these cited results may be linked to a new scheme of exact evolution of quantum $\phi^{4}$ field theory in $1+1$ dimensions. What is presented has a very strong convergence with the slope of graphs of electron-positron pair production representations. Furthermore, the similarities in plot behavior of the current values after the threshold electric field values argue in favor of the Bardeen pinning gap paradigm. We buttress these competing requirements by a new formulation given by Fred Cooper, et al., [3] [4] which will give more precise controls over the phase transitions inherent in the fate of the false vacuum hypothesis as brought up in our initial results and earlier publications. This leads to write the Gaussian wavefunctional like [1] [2].

$$
\Psi \equiv c \cdot \exp \left(-\alpha \cdot \int \mathrm{d} x\left[\phi-\phi_{C}\right]^{2}\right)
$$

Making this step from Equation (1) to Equation (2) involves recognizing, when we go to one-dimension, that we look at a given washboard potential with pinning energy contribution from $D \cdot \omega_{P}^{2}$ in one-dimensional CDW systems [1] [2].

$$
\frac{1}{2} \cdot D \cdot \omega_{P}^{2} \cdot(1-\cos \phi) \approx \frac{1}{2} \cdot D \cdot \omega_{P}^{2} \cdot\left(\frac{\phi^{2}}{2}-\frac{\phi^{4}}{24}\right)
$$

The fourth-order phase term is relatively small, so we look instead at contributions from the quadratic term and treat the fourth order term as a small perturbing contribution to get our one dimensional CDW potential, for the lowest order, to roughly look like Equation (2). In addition, we should note that the $c$ is due to an error functional-norming procedure, discussed below; $\alpha$ is proportional to one over the length of distance between instaton centers. This leads to [1] [2].

$$
c_{1} \cdot \exp \left(-\alpha_{1} \cdot \int \mathrm{d} \tilde{x}\left[\phi_{F}\right]^{2}\right) \cong \Psi_{\text {initial }}
$$

As well as

$$
c_{2} \cdot \exp \left(-\alpha_{2} \cdot \int \mathrm{d} \tilde{x}\left[\phi_{T}\right]^{2}\right) \cong \Psi_{\text {final }}
$$

The tunneling Hamiltonian incorporates wave-functionals whose Gaussian shape keeps much of the structure as represented by Fig. Following the false vacuum hypothesis, we have a false vacuum phase value $\phi_{F} \equiv\langle\phi\rangle_{1} \cong$ very small value, as well as having in $\mathrm{CDW}$, a final true vacuum $\phi_{T} \cong \varphi_{2 \pi} \equiv 2 \cdot \pi+\varepsilon^{+}$. This led to Gaussian wave-functionals with a simplified structure. For experimental reasons, we need to have (if we set the charge as equal to unity, as dimensionally speaking) [1] [2].

$$
\alpha \approx L^{-1} \equiv \Delta E_{\text {gap }} \equiv V_{E}\left(\phi_{F}\right)-V_{E}\left(\phi_{T}\right)
$$

From here on, we will be looking at the application of the above equation which is really using the fate of the false vacuum hypothesis.

\section{Representation of the Situation of Figure 1 below}

This situation as represented by Figure 1 eventually leads to a current along the lines of [1] [2].

$$
I \propto \tilde{C}_{1} \cdot\left[\cosh \left[\sqrt{\frac{2 \cdot E}{E_{T} \cdot c_{V}}}-\sqrt{\frac{E_{T} \cdot c_{V}}{E}}\right]\right] \cdot \exp \left(-\frac{E_{T} \cdot c_{V}}{E}\right)
$$

The current expression is a great improvement upon the phenomenological Zener current expression, where $G_{P}$ is the limiting Charge Density Wave (CDW) conductance [1] [2].

$$
\begin{array}{cr}
I \propto G_{P} \cdot\left(E-E_{T}\right) \cdot \exp \left(-\frac{E_{T}}{E}\right) & \text { if } E>E_{T} \\
0 & \text { otherwise }
\end{array}
$$


Figure 2 illustrates to how the pinning gap calculation improve upon a phenomenological curve fitting result used to match experimental data. The most important feature here is that the theoretical equation takes care of the null values before thre threshold is reached by itself, i.e. we do not need to set it to zero as is done arbitrarily in Equation (7).

So then, we have $L \propto E^{-1}$. When we consider a Zener diagram of CDW electrons with tunneling only happening when $e^{*} \cdot E \cdot L>\varepsilon_{G}$ where $e^{*}$ is the effective charge of each condensed electron and $\varepsilon_{G}$ being a pinning gap energy, we find, assuming that $\mathrm{x}$ is the de facto distance between an instanton pair and a measuring device. See references [1] [2].

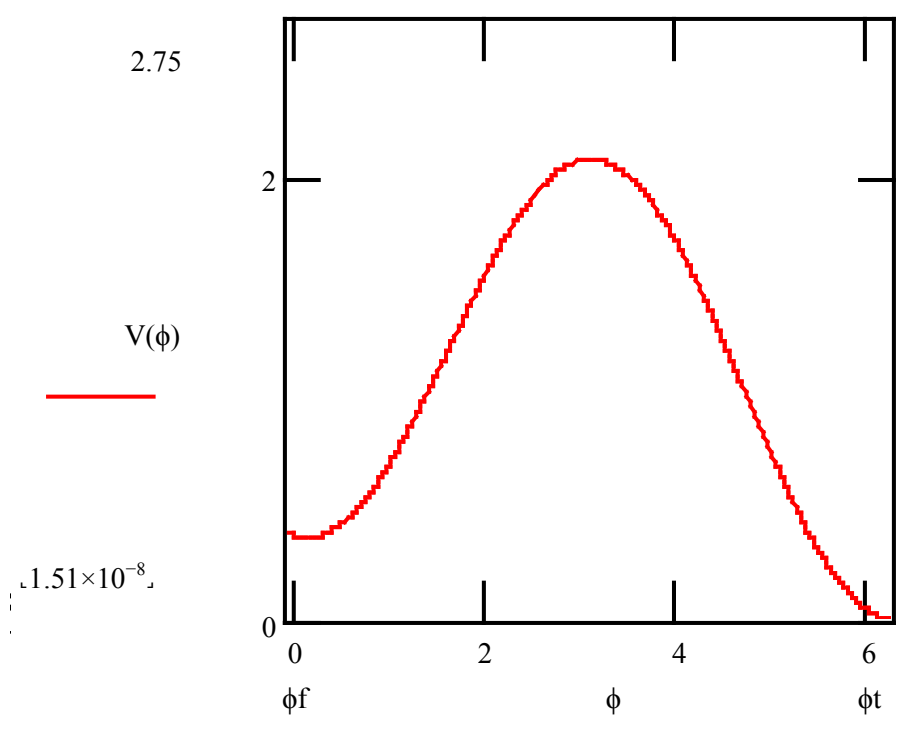

Figure 1. Fate of the false vacuum representation of what happens in CDW. This shows how we have a difference in energy between false and true vacuum values. See references [1] [2].

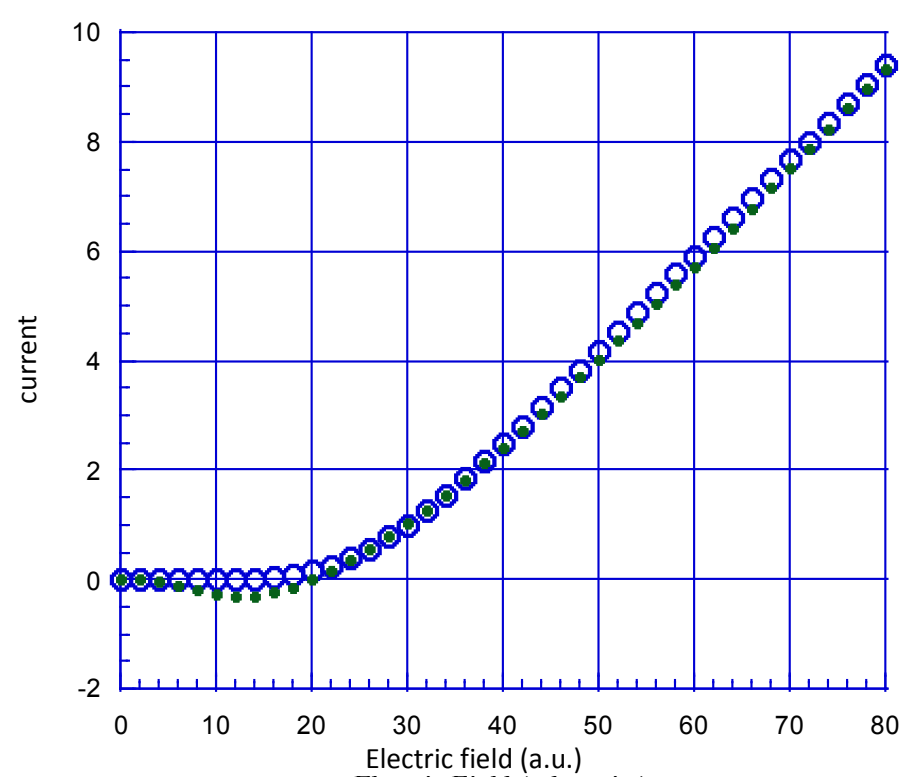

Figure 2. Experimental and theoretical predictions of current values versus applied electric field. The dots represent a Zenier curve fitting polynomial, whereas the blue circles are for the S-S' transport expression derived with a field theoretic version of a tunneling Hamiltonian. See references [1] [2]. 
In the current vs. applied electric field derivation results, we identify the $\Psi_{i}[\phi]$ as the initial wave function at the left side of a barrier and $\Psi_{f}[\phi]$ as the final wave function at the right side of a barrier [1] [2].

$$
\frac{L}{x} \cong c_{v} \cdot \frac{E_{T}}{E}
$$

\section{Conclusion and Linkage to Exact Dynamics of $\phi^{4}$ Field Theory in $1+1$ Dimensions}

We restrict this analysis to ultra fast transitions of CDW; this is realistic and in sync with how the wave-functionals used are formed in part by the fate of the false vacuum hypothesis. A kink-anti kink structure so implied by the Gaussian wave functional is stated by Cooper, and quoted by Moncrief [3] to have an evolution given by a sympletic evolution equation, as given below. Assuming an averaging procedure we can write as [3] [4].

$$
y_{i} \sim \int_{V_{i}} \mathrm{~d} x \phi[x, t] / \Delta V_{i} \approx \text { average of } \phi[x, t] \text { in a ball about } x_{i} \text { of volume } \Delta V_{i}
$$

And

$$
\frac{\mathrm{d} y_{i}}{\mathrm{~d} t} \equiv \pi_{i}[t]
$$

And

$$
\frac{\mathrm{d} \pi_{i}}{\mathrm{~d} t} \equiv \frac{1}{a^{2}} \cdot\left[y_{i+1}+y_{i-1}-2 y_{i}\right]-\lambda y_{i}^{3}+m^{2} y_{i}=F\left[y_{i}\right]
$$

This is assuming that we spatially discretize a Hamiltonian density via

$$
\int \mathrm{d} x \rightarrow a \cdot \sum_{i}
$$

Following a field theory replacement of $\hat{x} \rightarrow \varphi_{o p}[x, t]$, and a discretized time structure given by setting $t=j \in$, this leads to the possibility of looking at a quantum foam evolution as given in Figure 1 via the following sympletic structures, with $i$ the "spatial component along a chain", and $j$ the "time component" along a chain. Equation (13) and Equation (14) below are materially not different from having energy course through a wave lattice as seen in ocean swells accommodating an energy pulse through the water.

$$
\begin{aligned}
& y_{i}[j+1]=y_{i}[j]+\epsilon \cdot \pi_{i}[j]+\frac{\epsilon^{2}}{2} \cdot F\left(y_{i}[j]\right) \\
& \pi_{i}[j+1]=\pi_{i}[j]+\frac{\epsilon}{2} \cdot\left(F\left(y_{i}[j]\right)+F\left(y_{i+1}[j]\right)\right)
\end{aligned}
$$

A proper understanding of this evolution dynamic should permit a more mature quantum foam interpretation of false vacuum nucleation. The perturbations are outlined in Equation (13) and Equation (14) [3] [4]. Specifically, the expression for the net discretized force $F(y[j])$ above, when gone into, it will give details of why Figure 2 above holds, i.e. why the negative values which are so unphysical are cancelled out, giving more space-time structure as to Equation (6) above which the author derived in 2001.

\section{Acknowledgements}

The author thanks Dr. Yang Xi whom imbued the author with the background in the U of Houston to obtain a doctorate in physics also, my recently deceased father is thanked. This work is supported in part by National Nature Science Foundation of China grant No. 11375279.

\section{References}

[1] Beckwith, A.W. (2006) An Open Question: Are Topological Arguments Helpful in Setting Initial Conditions for Transport Problems in Condensed Matter physics? Modern Physics Letters B, 20, 233-243. 
http://arxiv.org/abs/math-ph/0411031

[2] Beckwith, A.W. (2006) A New S-S' Pair Creation Rate Expression Improving Upon Zener Curves for I-E Plots. Modern Physics Letters B, 20, 849-861. http://arxiv.org/abs/math-ph/0411045 http://dx.doi.org/10.1142/S0217984906011219

[3] Moncrief, V. (1983) Finite-Difference Approach to Solving Operator Equations of Motion in Quantum Theory. Physical Review D, 28, 2485. http://dx.doi.org/10.1103/PhysRevD.28.2485

[4] Sveshnikov, K.A. (1990) Finite-Difference Effects in Quantum Field Theory and Quantization of Classical Solutions. Theoretical and Mathematical Physics, 82, 37-45. http://dx.doi.org/10.1007/BF01028250 